\title{
Social and Nonsocial Autism Symptom Domains in Children and Adolescents with Autism Spectrum Disorder and Attention-Deficit/ Hyperactivity Disorder: Insights into Their Symptomatological Interplay
}

\author{
Judith T. Mack ${ }^{a}$ Nicole Wolffa Gregor Kohls $^{\mathrm{a}} \quad$ Andreas Becker ${ }^{\mathrm{b}}$ Sanna Stroth ${ }^{\mathrm{c}}$ \\ Luise Poustka $^{b}$ Inge Kamp-Becker ${ }^{c}$ Veit Roessner ${ }^{a}$ \\ aDepartment of Child and Adolescent Psychiatry, Faculty of Medicine, TU Dresden, Dresden, Germany; ${ }^{\text {b Department }}$ \\ of Child and Adolescent Psychiatry/Psychotherapy, University Medical Center Göttingen, Göttingen, Germany; \\ 'Department of Child and Adolescent Psychiatry, Psychosomatics and Psychotherapy, Medical Clinic, Philipps- \\ University Marburg, Marburg, Germany
}

\begin{abstract}
Keywords
Co-occurrence · Autism spectrum disorder · Attentiondeficit/hyperactivity disorder - Autism Diagnostic Interview-Revised · Autism Diagnostic Observation Schedule $\cdot$ Differential diagnosis
\end{abstract}

\begin{abstract}
Introduction: Autism spectrum disorder (ASD) and attention-deficit/hyperactivity disorder (ADHD) share overlapping symptomatology, particularly with regard to social impairments (including peer relationship difficulties), and they frequently co-occur. However, the nature of their co-occurrence remains unclear. Therefore, the current study aimed to examine the nature of the transdiagnostic link between ASD and ADHD from a symptomatological point of view measured with the Autism Diagnostic Observation Schedule (ADOS Module 3) and the Autism Diagnostic Interview-Revised (ADI-R). Methods: We analyzed the social and nonsocial ASD symptom domain scores from both diagnostic instruments in 4 clinically referred groups (i.e., ASD, ADHD,
\end{abstract}

ASD + ADHD, and no psychiatric diagnosis) without other co-occurring mental disorders using a two-by-two full-factorial MANOVA design with the factors ASD (yes/no) and ADHD (yes/no). Results: We found no ASD by ADHD interaction effects across all symptom domain scores of ADOS and ADI-R, except for ADOS imagination/creativity. There were only main effects of the factor ASD but no main effects of ADHD. Follow-up contrasts showed that exclusively, ASD had an impact on the measured symptomatology in case of co-occurring ASD + ADHD. Conclusion: Overall, the results support an additive model of the symptomatology across areas of communication, social interaction, and stereotyped behaviors and restricted interests in case of the co-occurrence of ASD and ADHD when assessed with ADOS/ADI-R. Thus, one can assume that the phenotypic overlap of ASD + ADHD may be less complicated than suspected - at least with regard to ASD symptomatology - and that in the presence of ADHD, ASD symptomatology is generally well measurable with best-practice diagnostic instruments.

(C) 2021 The Author(s)

Published by S. Karger AG, Basel karger@karger.com www.karger.com/psp

Karger $\stackrel{\text { ' }}{5}$

BOPEN ACCESS
(C) 2021 The Author(s)

Published by S. Karger AG, Basel

This article is licensed under the Creative Commons Attribution 4.0 International License (CC BY) (http://www.karger.com/Services/ OpenAccessLicense). Usage, derivative works and distribution are permitted provided that proper credit is given to the author and the original publisher.
Correspondence to:

Gregor Kohls, gregor.kohls@ uniklinikum-dresden.de 


\section{Introduction}

Autism spectrum disorder (ASD) and attention-deficit/hyperactivity disorder (ADHD) are common, severely impairing neurodevelopmental disorders which typically onset in early childhood. Although the diagnostic criteria for the 2 disorders clearly point to distinct core symptom sets [1], diagnoses of ASD and ADHD frequently co-occur, and research suggests that there is overlap at the phenotypic level, for instance in clinical symptomatology that is shared by ASD and ADHD [2-4]. Specifically, while social impairments are a hallmark of individuals with ASD, children with ADHD also often exhibit social difficulties that resemble those seen in ASD, such as problems in relating to other people or inappropriate peer-related behaviors [5-10]. Consequently, differentiating between ASD and ADHD is apparently not a trivial undertaking in clinical practice, and the same applies to correctly classifying the co-occurrence of both disorders within the same individual.

Several studies and reviews have focused on the nature of the transdiagnostic link between the 2 conditions from different research perspectives, including genetics, cognition, neurobiology, and clinical symptomatology, but results and hence conclusions are rather mixed [11-13]. In terms of symptomatology, previous research showed that individuals with ASD + ADHD display greater ASD symptom severity than individuals with ASD or ADHD when simple pairwise comparisons are used [14-17]. However, other studies did not find such differences between individuals with ASD + ADHD and for instance those with ASD $[10,18]$. Moreover, children with ADHD often present with elevated ASD ratings, particularly with regard to social communication difficulties $[19,20]$, even to the extent that many individuals could be false-positively categorized as ASD using the Autism Diagnostic Observation Schedule (ADOS) [21] and the Autism Diagnostic Interview-Revised (ADI-R) [22, 23] .

Inconsistent findings across studies might be explained by differences in sample composition (e.g., age range, IQ level, gender ratio, or the presence of additional co-occurring mental disorders) or diagnostic procedures used (e.g., DSM vs. International Classification of Diseases [ICD], varying assessment instruments, and categorical vs. dimensional approach). However, they may also reflect methodological shortcomings, such as using only pairwise group comparisons (i.e., ASD vs. ASD + $\mathrm{ADHD}$ vs. $\mathrm{ADHD}$ ) when investigating the co-occurrence of both conditions [24]. To overcome such shortcoming, it is necessary to apply appropriate statistical testing in the

Autism Symptomatology in ASD versus ADHD form of a full-factorial design with 4 groups (ASD, ADHD, ASD + ADHD, and controls) enabling computations based on the factors ASD (yes/no) and ADHD (yes/no) and their potential interaction. To our knowledge, this has been done to date only by Tye and colleagues for investigating different cognitive functions [25-28], including emotional face and gaze processing, but not yet in terms of clinical ASD symptomatology.

Within a full-factorial design, the lack of an interaction effect between the 2 factors ASD and ADHD would support an additive model in which the co-occurrence of both diagnoses (i.e., ASD + ADHD) simply represents a combination of the 2 distinct pathologies. Such model suggests that the symptomatology of patients with ASD + ADHD is impacted by symptoms of ASD in addition to symptoms of $\mathrm{ADHD}$ (or vice versa) in a summative manner (for further explanation of additive effects see [29, 30]). By contrast, an interactive model (i.e., the presence of an interaction effect) would suggest that the co-occurrence of both diagnoses represents a separate nosological entity. In case that an additive model is supported, the relative contribution of the 2 factors ASD and ADHD to the symptomatology in the ASD + ADHD group could be further determined by follow-up contrasts [31].

Thus, the current study aimed at investigating social and nonsocial clinical symptomatology in 4 groups (i.e., ASD, ADHD, ASD + ADHD, and no psychiatric diagnosis [ND]) without other co-occurring mental disorders using a two-by-two full-factorial design with the factors ASD (yes/no) and ADHD (yes/no). Here, we focused on core ASD symptom domains since they are the most challenging in the differential and co-occurring diagnostic process [1], particularly the social difficulties seen in both groups (ASD and ADHD). More specifically, we aimed to clarify (i) whether the social and nonsocial clinical symptomatology assessed with best-practice ASD-specific diagnostic instruments (i.e., ADOS and ADI-R) fits with an additive or interactive model of the co-occurrence of ASD + ADHD and (ii) in case that an additive model is supported, which factor(s) (i.e., ASD and/or ADHD diagnosis) is/are related to the symptomatology scores on these instruments. Additionally, we calculated contrasts to (iii) specify the relative impact of the factor ASD and/or ADHD by direct comparison, e.g., the factor ASD adds more to the different ADOS/ADI-R domain scores than the factor ADHD. Considering the recent literature review on the topic by Antshel and Russo [11] and the work by Tye et al. [25-28], we expected to find an "additive" profile regarding ASD symptomatology in case of the cooccurrence of ASD and ADHD. 
Table 1. Sample description

\begin{tabular}{|c|c|c|c|c|c|c|}
\hline \multirow[b]{2}{*}{ Age } & \multirow[t]{2}{*}{$\operatorname{ASD}(n=174)$} & \multirow[t]{2}{*}{$\operatorname{ADHD}(n=37)$} & \multirow[t]{2}{*}{$\mathrm{ASD}+\mathrm{ADHD}(n=30)$} & \multirow[t]{2}{*}{$\mathrm{ND}(n=33)$} & \multicolumn{2}{|c|}{ Group differences } \\
\hline & & & & & $F(3,270)$ & $p$ value \\
\hline$M(S D)$ & $10.45(2.59)$ & $9.68(2.29)$ & $10.00(2.03)$ & $9.61(2.33)$ & 1.880 & 0.133 \\
\hline Min-max & $5-17$ & $6-15$ & $6-14$ & $6-15$ & & \\
\hline Gender, \% & & & & & $x^{2}(d f=3)$ & $p$ value \\
\hline Female & 9.2 & 2.7 & 16.7 & 12.1 & 4.067 & 0.254 \\
\hline Male & 90.8 & 97.3 & 83.3 & 87.9 & & \\
\hline $\mathrm{IQ}^{*}$ & & & & & $F(3,233)$ & $p$ value \\
\hline$n$ & 154 & 29 & 25 & 29 & 0.468 & 0.705 \\
\hline$M(S D)$ & $100.92(19.08)$ & $101.86(19.34)$ & $96.68(17.78)$ & 99.24 (15.93) & & \\
\hline Min-max & $55-143$ & $72-134$ & $58-124$ & $58-135$ & & \\
\hline
\end{tabular}

$N($ total $)=274$. ASD, autism spectrum disorder; ADHD, attention-deficit/hyperactivity disorder; ASD + ADHD, co-occurrence of both diagnoses; ND, no psychiatric diagnosis; M, mean; SD, standard deviation; $n$, sample size; ID, intellectual disability. ${ }^{*}$ Considering solely an IQ criterion of $<70$ as a cutoff for ID, there was the following proportion of ID per group: ADHD (2.8\%), ASD (3.8\%), ASD + ADHD (11.5\%), and ND (3.4\%), with no significant differences between groups $(p=0.29)$.

\section{Materials and Methods}

\section{Sample}

Our sample, including the youth without a psychiatric diagnosis (ND), was derived from a large database of individuals referred to specialized outpatient clinics for children and adolescents and/ or adults with ASD. The database was created by 4 of the leading ASD research groups in Germany (Marburg, Dresden, Berlin, and Mannheim) as part of the ASD-Net consortium [32]. It includes 2,453 patients (age in years: $M=13.56 \pm 10.61$; $\min =18$ months; $\max =72$ years; $16.8 \%$ female), who were referred with suspected ASD to one of the 4 outpatient clinics. All participants were diagnosed according to the ICD-10 [33], using "gold-standard" bestestimate clinical (BEC) diagnoses [23]. BEC diagnosis was determined by at least 2 experienced clinicians from a multidisciplinary team (which included psychologists and/or psychiatrists) after extensive examination and review of all available information from a patient's medical record. Medical records included IQ, neuropsychological testing, questionnaires, reports from other institutions, school reports, home videos, ADOS [21], ADI-R [22], and a differential/co-occurring diagnoses algorithm performed by an experienced clinician. Both ADOS and ADI-R were conducted by clinically trained team members at each center who were all licensed to do so.

For the purpose of the current study, our sample was selected according to the following criteria: (i) referred for a clinical ASD diagnostic assessment, (ii) complete data of ADOS Module 3 (ADOS-M3) and ADI-R, (iii) age of 4-18 years, (iv) verbally fluent, (v) BEC diagnosis of ASD (F84.0, F84.1, or F84.5) but no ADHD, (vi) BEC diagnosis of ADHD (all subtypes F90.0 or F98.8) but no $\mathrm{ASD}$, (vii) co-occurring ASD + ADHD, and (viii) no psychiatric diagnosis (ND). The patients with ASD, ADHD, and ASD + ADHD had no other psychiatric diagnoses. To keep the sample representative of the common population in ASD specialty clinics, there were no exclusion criteria regarding IQ or gender. This resulted in a sample of $n=274$ individuals (age in years: $M=10.20$ $\pm 2.48, \min =5, \max =17 ; 10 \%$ female), including $n=174$ patients with ASD, $n=37$ with ADHD, $n=30$ with ASD + ADHD, and $n=33$ with ND. The 4 groups did not differ in age $(p=0.133)$, IQ $(p=0.705)$, or gender distribution $(p=0.254$; see Table 1$)$.

\section{Measures}

Our analyses included the domain scores of the so-called goldstandard instruments in diagnosing ASD: ADOS [21,34] and ADI-R $[35,36]$. Both are based on ASD criteria of ICD-10 [33] and DSM-IV-TR [37] and can be used to obtain information about ASD symptoms across different behavioral domains.

The ADOS is a well-established, standardized, semi-structured observational instrument. It consists of 4 modules whose application depends on the patient's expressive language and developmental levels. The current analyses focused on ADOS-M3, which is used for verbally fluent children and adolescents, and it evaluates language and communication, reciprocal social interaction (SI), play/imagination, and stereotyped behaviors and restricted interests (RRB). The ADOS-M3 contains a set of standardized scenarios that an ADOS-trained clinician walks through with the patient. Afterward, the patient's behavior is rated on 28 items. These item codes are converted according to the ADOS manual so that each item is rated on a 3 -point scale from $0=$ no abnormality to $2=$ moderate to severe abnormality. The ADOS-M3 yields 4 domain scores, which are the sum of certain of the 28 items: communication (Com), SI, imagination/creativity, RRB, and a total score (sum of the domain scores Com and SI) [21, 34, 38]. Other abnormal behaviors (section $\mathrm{E}$ of the ADOS-M3) were not included as no domain score can be built. For the German version [38] found adequate internal consistency, sensitivity, and specificity.

The ADI-R is a semi-structured parent interview with 93 items on ASD symptoms covering lifetime and early-childhood behavior. The items are rated by a trained clinician and converted according to the ADI-R manual so that each item is rated on a 3-point scale from $0=$ no abnormality to $2=$ moderate to severe abnormality. The ADI-R yields 4 domain scores, created from subsets of the
10
Psychopathology 2023;56:8-16 DOI: $10.1159 / 000520957$
Mack/Wolff/Kohls/Becker/Stroth/ Poustka/Kamp-Becker/Roessner 
Table 2. ADOS-M3 $2 \times 2$ ANOVA for the factors ASD and ADHD

\begin{tabular}{|c|c|c|c|c|c|c|c|c|c|c|c|}
\hline \multirow[t]{2}{*}{ ADOS domains } & \multirow{2}{*}{$\begin{array}{l}\text { ASD } \\
(n=174) \\
M(S D)\end{array}$} & \multirow{2}{*}{$\begin{array}{l}\text { ADHD } \\
(n=37) \\
M(S D)\end{array}$} & \multirow{2}{*}{$\begin{array}{l}\text { ASD + ADHD } \\
(n=30) \\
M(S D)\end{array}$} & \multirow{2}{*}{$\begin{array}{l}\text { ND } \\
(n=33) \\
M(S D)\end{array}$} & \multicolumn{7}{|c|}{ ANOVA $F(1,270)$ with $\left(\eta^{2}\right)$} \\
\hline & & & & & ASD & & & ADHD & & $A S D \times A D$ & HD \\
\hline Com & $3.88(1.71)$ & $1.16(1.17)$ & $3.87(2.10)$ & $1.91(1.63)$ & 79.928 & $* * *$ & $(0.228)$ & 2.110 & $(0.008)$ & 1.972 & $(0.007)$ \\
\hline SI & $7.83(2.71)$ & $3.49(2.56)$ & $8.60(2.69)$ & $3.70(2.87)$ & 120.852 & $* * *$ & $(0.309)$ & 0.447 & $(0.002)$ & 1.366 & $(0.005)$ \\
\hline Total score (Com + SI) & $11.71(3.93)$ & $4.65(3.51)$ & $12.47(4.55)$ & $5.61(4.12)$ & 127.426 & $* * *$ & $(0.321)$ & 0.026 & $(0.000)$ & 1.939 & $(0.007)$ \\
\hline Imagination/creativity & $1.14(0.75)$ & $0.62(0.59)$ & $1.30(0.84)$ & $0.94(0.70)$ & 14.915 & $* * *$ & $(0.052)$ & 0.499 & $(0.002)$ & $4.303 *$ & $(0.016)$ \\
\hline RRB & $1.09(1.25)$ & $0.32(0.53)$ & $1.03(1.43)$ & $0.48(0.80)$ & 13.556 & $* * *$ & $(0.048)$ & 0.376 & $(0.001)$ & 0.081 & $(0.000)$ \\
\hline
\end{tabular}

Effects are from $2 \times 2$ ANOVA with ASD (yes/no) and ADHD (yes/no) as factors. ASD, autism spectrum disorder; ADHD, attention-deficit/hyperactivity disorder; ASD + ADHD, co-occurrence of both diagnoses; ND, no psychiatric diagnosis; RRB, stereotyped behaviors and restricted interests; M, mean; SD, standard deviation; $n$, sample size; $\eta^{2}$, eta square for effect size; SI, social interaction; Com, communication; ADOS-M3, Autism Diagnostic Observation Schedule Module 3. ${ }^{* * *} p<0.001 .{ }^{*} p<0.05$.

Table 3. ADOS-M3 - comparison of the main factors

\begin{tabular}{lllll}
\hline ADOS domains & Contrast score & $t(270)$ & & Factor comparison $^{\mathrm{a}}$ \\
\hline Com & 2.72 & 8.913 & $* * *$ & ASD > ADHD \\
SI & 4.34 & 8.854 & $* * *$ & ASD > ADHD \\
Total score (Com + SI) & 7.06 & 9.818 & $* * *$ & ASD > ADHD \\
Imagination/creativity & - & - & & - \\
RRB & 0.77 & 3.683 & $* * *$ & ASD > ADHD \\
\hline
\end{tabular}

ASD, autism spectrum disorder; ADHD, attention-deficit/hyperactivity disorder; ASD + ADHD, co-occurrence of both diagnoses; ND, no psychiatric diagnosis; RRB, stereotyped behaviors and restricted interests; SI, social interaction; Com, communication; ADOS-M3, Autism Diagnostic Observation Schedule Module 3. ${ }^{* * *} p<0.001$. ${ }^{\text {a }}$ The calculation was performed on the assumption that the difference in the sum of mean values from the ADOS subscales between children with or without ASD disorder does not deviate from the difference in the sum of mean values in children with or without ADHD ([ASD/ADHD + ASD] $-[\mathrm{ADHD}+$ Control $]=[\mathrm{ASD} / \mathrm{ADHD}+\mathrm{ADHD}]-[\mathrm{ASD}+$ control $])$. After solving the equations, the following contrast weights resulted: ND, 0; ASD, 1 ; ADHD, -1 and ASD/ADHD, 0 . ${ }^{b}$ Contrasts could not be computed due to the significant interaction in the ANOVA (Table 2).
93 items: Com, SI, RRB, and abnormality of development evident at or before 36 months of life. The last domain was not considered in this study due to many missing values. The interrater reliability of the domain SI was reported to be $r_{\mathrm{kw}}=0.75$; for Com, $r_{\mathrm{kw}}=0.77$; and for $R R B, r_{\mathrm{kw}}=0.80[38,39]$. The internal consistency was reported to be $\alpha=0.91$ for the domain SI, $\alpha=0.83$ for Com, and $\alpha=$ 0.64 for the domain $R R B$ [38].

\section{Data Analysis}

The domain scores of ADOS and ADI-R were analyzed using two-way MANOVAs, followed by univariate ANOVAs with the between-subject factors ASD (yes/no) and ADHD (yes/no). A possible additive model of ASD and ADHD would be supported by the absence of an interaction effect. In such case, contrasts were calculated to compare the contribution of the 2 factors to the ASD symptomatology when ASD and ADHD co-occur. The calculations were computed on the assumption that the difference in the sum of mean values from the ADOS and ADI-R domain scores between children and adolescents with or without ASD does not deviate from the difference in the sum of mean values in those with or without ADHD $([\mathrm{ASD}+\mathrm{ADHD}+\mathrm{ASD}]-[\mathrm{ADHD}+\mathrm{ND}]=$ $[\mathrm{ASD}+\mathrm{ADHD}+\mathrm{ADHD}]-[(\mathrm{ASD}+\mathrm{ND}])$. The equation results in the following contrast weights: $\mathrm{ND}=0 ; \mathrm{ASD}=1 ; \mathrm{ADHD}=-1$; and ASD $+\mathrm{ADHD}=0$ [31]. All analyses were computed with SPSS (IBM SPSS Statistics, Version 25) using a significance level of 5\%.

\section{Results}

\section{ADOS}

The $2 \times 2$ MANOVA revealed no overall interaction effect between the factors ASD (yes/no) and ADHD (yes/ no) across all 5 ADOS domain scores. There was however a main effect of $\operatorname{ASD}(F(4,267)=31.916 ; p<0.001$; 


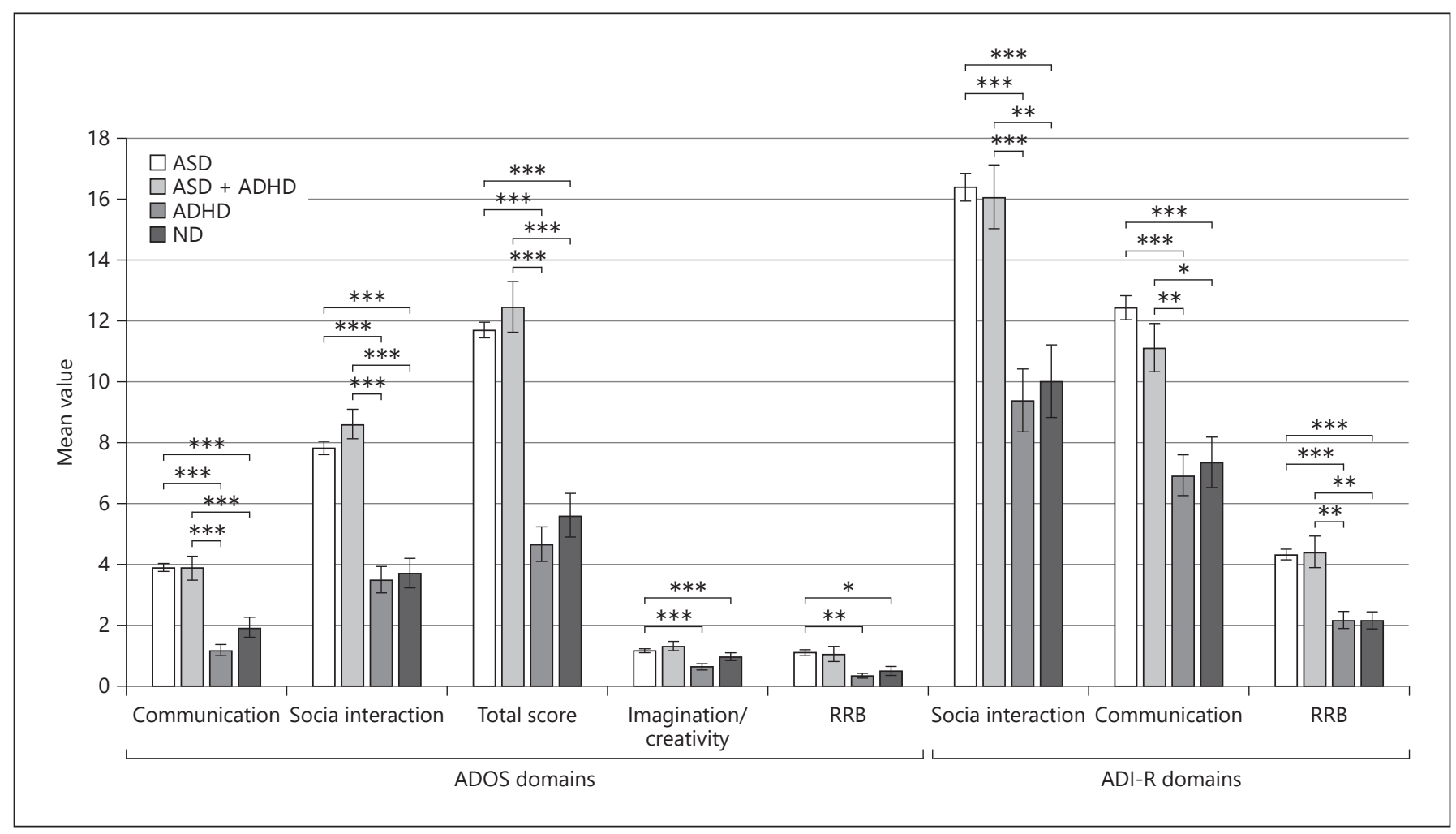

Fig. 1. ADOS and ADI-R symptom domain scores. See main text for results. Means and SEMs are given. ${ }^{* * *} p<$ $0.001 ;{ }^{* *} p<0.01 ;{ }^{*} p<0.05$. ASD, autism spectrum disorder; ADHD, attention-deficit/hyperactivity disorder; ASD + ADHD, co-occurrence of both diagnoses; ND, no psychiatric disorder; RRB, stereotyped behaviors and restricted interests; ADOS, Autism Diagnostic Observation Schedule; ADI-R, Autism Diagnostic Interview-Revised.

partial $\left.\eta^{2}=0.323\right)$ but not of ADHD $(p=0.140)$. Table 2 shows the follow-up $2 \times 2$ univariate ANOVAs, confirming the main effects for the factor ASD on each of the ADOS domains. As expected, no main effects were found for the factor ADHD (all $p>0.147$ ). To further specify the impact of ASD and/or ADHD on each of the ADOS domains, additional contrasts were calculated, except for the domain imagination/creativity due to the significant interaction effect $(p=0.039)$ which points to a lack of an additive model with regard to this symptom domain (Fig. 1). Note however that this interaction effect would not survive correction for multiple comparisons. Table 3 shows that the factor ASD had indeed stronger impact than that of ADHD on all ADOS domains.

\section{$A D I-R$}

The $2 \times 2$ MANOVA revealed no overall interaction effect between the factors ASD and ADHD across all ADI-R domain scores $(p=0.757)$. Similar to the ADOS domains, there was a main effect of $\operatorname{ASD}(F(3,268)=$ 20.088; $p<0.001$; partial $\left.\eta^{2}=0.184\right)$ but not of ADHD $(p=0.555)$. Table 4 shows the follow-up $2 \times 2$ univariate ANOVAs, confirming the main effects for the factor ASD on the 3 analyzed ADI-R domains. No effects for the factor ADHD (all $p>0.571$ ) or interaction effects (all $p>$ 0.253 ) were found (Table 4). The calculated contrasts showed again that the factor ASD was more strongly related to all ADI-R domains than the factor ADHD (Table 5).

\section{Discussion}

The main objective of this study was to determine the extent to which the co-occurrence of ASD and ADHD impacts on social and nonsocial clinical symptomatology as assessed with best-practice ASD-specific diagnostic instruments among clinically referred children and adoles-
12

Psychopathology 2023;56:8-16 DOI: $10.1159 / 000520957$
Mack/Wolff/Kohls/Becker/Stroth/ Poustka/Kamp-Becker/Roessner 
Table 4. ADI-R $2 \times 2$ ANOVA for the factors ASD and ADHD

\begin{tabular}{|c|c|c|c|c|c|c|c|c|c|c|c|}
\hline ADI-R domains & $\begin{array}{l}\text { ASD } \\
(n=174) \\
M(S D)\end{array}$ & $\begin{array}{l}\text { ADHD } \\
(n=37) \\
M(S D)\end{array}$ & $\begin{array}{l}\text { ASD + ADHD } \\
(n=30) \\
M(S D)\end{array}$ & $\begin{array}{l}\text { ND } \\
(n=33) \\
M(S D)\end{array}$ & ASD & & & ADHD & & ASD $\times$ & HD \\
\hline SI & $16.43(6.00)$ & $9.38(6.28)$ & $16.10(5.76)$ & 10.03 (6.88) & 47.685 & $* * *$ & $(0.150)$ & 0.268 & $(0.001)$ & 0.029 & $(0.000)$ \\
\hline Com & $12.45(5.27)$ & $6.92(4.12)$ & $11.13(4.27)$ & $7.36(4.72)$ & 36.390 & $* * *$ & (0.119) & 1.310 & $(0.005)$ & 0.323 & $(0.001)$ \\
\hline RRB & $4.31(2.47)$ & $2.16(1.68)$ & $4.40(2.87)$ & $2.15(1.62)$ & 36.586 & $* * *$ & $(0.119)$ & 0.019 & $(0.000)$ & 0.012 & $(0.000)$ \\
\hline
\end{tabular}

Effects are from $2 \times 2$ ANOVA with ASD (yes/no) and ADHD (yes/no) as factors. ASD, autism spectrum disorder; ADHD, attention-deficit/ hyperactivity disorder; ASD + ADHD, co-occurrence of both diagnoses; ND, no psychiatric diagnosis; RRB, stereotyped behaviors and restricted interests; $M$, mean; $S D$, standard deviation; $n$, sample size; $\eta^{2}$, eta square for effect size; ADI-R, Autism Diagnostic Interview-Revised; $\mathrm{SI}$, social interaction; Com, communication. ${ }^{* * *} p<0.001$.

Table 5. ADI-R - comparison of the main factors

\begin{tabular}{llll}
\hline ADI-R domains & Contrast score & $t(270)$ & $\begin{array}{l}\text { Factor } \\
\text { comparison }\end{array}$ \\
\hline SI & 7.05 & $6.365 * * *$ & ASD $>$ ADHD \\
Com & 5.54 & $6.155^{* * *}$ & ASD $>$ ADHD \\
RRB & 2.15 & $5.069 * * *$ & ASD $>$ ADHD \\
\hline
\end{tabular}

ASD, autism spectrum disorder; ADHD, attention-deficit/ hyperactivity disorder; ASD + ADHD, co-occurrence of both diagnoses; ND, no psychiatric diagnosis; RRB, stereotyped behaviors and restricted interests; ADI-R, Autism Diagnostic Interview-Revised; $\mathrm{SI}$, social interaction; Com, communication. ${ }^{* * *} p<0.001$. ${ }^{\mathrm{a}}$ The calculation was performed on the assumption that the difference in the sum of mean values from the ADOS subscales between children with or without ASD disorder does not deviate from the difference in the sum of mean values in children with or without ADHD ([ASD/ $\mathrm{ADHD}+\mathrm{ASD}]-[\mathrm{ADHD}+$ Control $]=[\mathrm{ASD} / \mathrm{ADHD}+\mathrm{ADHD}]-[\mathrm{ASD}+$ control]). After solving the equations, the following contrast weights resulted: ND, 0; ASD, 1; ADHD, -1; and ASD/ADHD, 0.

cents using a full-factorial group design. To do so, we assessed and compared children and adolescents of 4 groups (i.e., ASD, ADHD, ASD + ADHD, and ND) who had no additional psychiatric diagnoses. While the groups did not differ in age, gender, and IQ, they differed in their expression across most of the ASD symptom domains measured with ADOS and ADI-R. Notably, we found no interaction effect for the factors ASD and ADHD, except for the domain imagination/creativity on the ADOS. Thus, overall, our findings would rather support an additive model for ASD-like symptomatology in case of the co-occurrence of ASD and ADHD.

Varieties of models exist to explain the co-occurrence of psychiatric disorders, such as ASD + ADHD, but only very few of such models have been sufficiently tested so far [29]. Therefore, existing findings have led some authors to suggest that it might be near impossible to determine whether ASD symptoms in ADHD represent ADHD, co-occurring ASD or even a completely separate disorder [40,41]. However, a recent literature review on the topic concludes that the co-occurrence of ASD and ADHD indicates an "additive" profile of the 2 conditions [11]. Interestingly, the review did not include the work by Tye and colleagues who used a two-by-two factorial design (ASD yes/no; ADHD yes/no) in a small sample of boys (8-13 years) investigating face/gaze processing [27], emotion processing [28], executive functions [25], and response time variability [26]. While the authors found distinct abnormalities for ASD as well as ADHD across these different cognitive domains, they did not reveal any significant interaction effects between ADHD and ASD diagnosis on these measures, suggesting an additive cooccurrence of the unique disorders. Thus, our phenotypic data corroborate and extend the existing literature in support of an additive model regarding ASD-like symptomatology in children and adolescents with ASD + ADHD.

The follow-up contrasts that we calculated to specify the impact of the factor ASD and the factor ADHD in case of co-occurrence showed that exclusively the factor ASD contributed to the clinical symptomatology assessed by ADOS and ADI-R. The factor ADHD had no impact. That is not entirely surprising given that ADOS and ADI$\mathrm{R}$ have been developed specifically for assessing ASD symptomatology, and this should result in higher symptom scores among individuals with ASD than those with ADHD. However, previous studies have clearly shown that ADHD groups may exhibit ASD-like symptoms and SI and Com impairments, like peer relationship problems 
[4], and they could even meet ASD cutoffs on the ADOS and ADI-R [23]. The other way around, a co-occurring ADHD diagnosis or elevated ADHD-like symptoms can aggravate maladaptive behavior in individuals with ASD, resulting in a higher autistic trait and externalizing problems [17]. Taken together, these findings highlight that social difficulties are not unique to ASD but should also be recognized as being part of the ADHD condition. Interestingly, a recent meta-analysis suggests that diagnostic subtypes of ADHD may differ with regard to their social difficulties [42]. While children with the combined type of ADHD are significantly more impaired than groups with predominantly inattentive or hyperactive/ impulsive symptoms in overall social functioning, prosocial behavior, and peer relationships (i.e., disliked by others), children with the inattentive type show higher levels of shy and passive social behavior than the combined type.

The finding of higher ASD symptom domain scores in both groups with ASD (ASD and ASD + ADHD) than those in the 2 groups without ASD (ADHD and ND) is in line with some of the previous findings that are based on pairwise group comparisons [10,23]. However, results on (no) differences regarding ASD symptomatology between ASD and ASD + ADHD are still rather mixed. In line with our findings here, Yerys and colleagues [17], for example, found no phenotypic differences between ASD and ASD + ADHD when measured with ADOS and ADIR. Yet their groups differed on other instruments, such as the Social Responsiveness Scale, with the ASD + ADHD group having more ASD symptoms than those of the pure ASD group [17]. Others found higher ASD severity scores in individuals with ASD + ADHD than those with ASD across different domains measured with the ADI-R but not with ADOS $[10,15,16]$. These inconsistent findings are likely caused by not only the heterogeneous nature of clinical samples in general but also by various study-related differences, like inclusion/exclusion of (other) cooccurring disorders, definition of ADHD status (e.g., elevated symptoms vs. clinical diagnosis), patients' age range, or ASD assessment instruments used (e.g., screening questionnaires vs. ADOS/ADI-R, incl. different ADOS modules). This highlights once again that methodological differences and potential shortcomings clearly limit the comparability between studies and thus replication of findings, precluding firm conclusions.

The findings of the current study should be interpreted in light of some limitations. First, while our study sample stems from a large database of individuals referred to specialized outpatient clinics for ASD, we were only able to include relatively few female patients. Although the prevalence of ASD and ADHD is considerably lower in girls than boys, the present findings might not fully apply to female patients. Thus, future work should focus more strongly on differences and similarities in symptom expression in ASD, ADHD, and the co-occurrence of both conditions, specifically in female patients who are still underrepresented in this line of research [43]. Second, our ND group was recruited via the outpatient clinics too, which may have biased our findings to some extent. Notably, as shown in Figure 1, the ND group was not entirely free of ASD symptoms and scored at least numerically higher on most of the ASD symptom domains than the ADHD group. As such, this group is not representative for typically developing children. Third, we restricted our analyses to participants who completed the ADOSM3 (i.e., nonverbal individuals were not included) and who were free of other co-occurring psychiatric diagnoses. Thus, our sample was deliberately preselected, which limits its representativeness. Fourth, it has to be considered that the present data are based on the ADOS but not the more recent ADOS-2 manual [44]. This is because data collection spanned across a relatively long time period, dating back to when the German version of the ADOS- 2 was not yet available. Therefore, our results cannot easily be generalized to DSM-5 criteria. Fifth, we did not include ADHD symptoms in our analyses that are considered to be core to the ADHD diagnosis as ADHDrelated clinical data were not available for the entire sample. Thus, follow-up studies should preferably utilize additional assessment instruments for ADHD-specific symptomatology and also extend the research focus on other relevant (e.g., etiological) factors, including neurobiological and neurocognitive parameters [13], as well as investigate different ADHD subtypes [42]. It should also be noted that our ADHD group represents a preselected and less representative subsample of patients with ADHD as this group consisted of children who were seen in outpatient clinics for ASD due to ASD concerns by their parents. The data for the present study were obtained during routine clinical evaluations at ASD specialty clinics using "gold-standard" ASD diagnostic instruments, such as ADOS and ADI-R. In this context, the co-occurrence of ASD and ADHD arises particularly frequently and in many complex cases. Using ADOS and ADI-R, clinicians are able to detect ASD-like symptomatology in ADHD patients, including social communication and peer relationship problems [23]; therefore, this assessment approach is of high ecological and economic advantage as it is a pragmatic one. Moreover, since we recruited a clinical 
rather than a research population, this study sample is unique and likely most relevant to practitioners. Nevertheless, the symptomatological data at hand provide only information at the on-average group level but is less informative for the (differential) diagnostic process on an individual case-by-case basis, which would be ideal in the context of personalized medicine.

In conclusion, the current study investigated the cooccurrence of ASD and ADHD from a symptomatological perspective measured with the "gold-standard" instruments for assessing ASD in clinical practice. We provide further evidence in support of an additive model for this co-occurrence with regard to the core ASD symptom domains. Hence, clinically, ASD + ADHD should not be seen necessarily as a separate subgroup or subtype, and therefore, one can assume that the phenotypic overlap in the ASD + ADHD group is probably less complicated than suspected. As mentioned above, while even in the presence of ADHD, ASD-like symptoms, including social deficits, are on average well measurable with ADOS and ADI-R; ADHD (co-occurring or differential) should still be evaluated with ADHD-specific instruments. Notably, individuals referred to outpatient clinics specialized for ASD are not routinely screened for ADHD as part of the evaluation procedure, which is largely due to pragmatic considerations (e.g., financial and personnel resources or time resources of patient and families). Therefore, one can contemplate developing an ADHD screening score based on ADOS/ADI-R to guide the decision-making about the additional effort of evaluating a patient also for ADHD not only clinically but also data-driven. Finally, the sensitivity and specificity of both ADOS and ADI-R for diagnosing ASD in case of maximal bias due to cooccurring ADHD should be examined to substantiate our findings from another perspective.

\section{Statement of Ethics}

The study was carried out in accordance with the recommendations of good clinical practice and in accordance with the Declaration of Helsinki and national legislation. This study protocol was reviewed and approved by the Ethics Committee of the Charité Berlin, approval number EA4/129/19. Written informed consent was not required for this study.

\section{Conflict of Interest Statement}

The authors have no conflicts of interest to declare.

\section{Funding Sources}

This research was funded by the German Federal Ministry of Education and Research (BMBF, grant No.: FKZ 01 EE1409A). The funding source had no role in the study design, collection, analysis, interpretation of data, writing of the article, or decision to submit the article for publication.

\section{Author Contributions}

J.T.M. analyzed the data, prepared the tables and figures, and drafted the manuscript. V.R. and A.B. conceived the study, revised the manuscript, and assisted in statistical methodology. N.W., S.S., L.P., and I.K.-B. provided data for the study and revised the manuscript. G.K. contributed to revising the manuscript. All the authors read, commented on drafts of the paper, and approved the final manuscript and its submission.

\section{Data Availability Statement}

The datasets generated for this study are available on reasonable request in line with the data sharing policy of the ASD-Net consortium.

\section{References}

1 American Psychiatric Association. Diagnostic and statistical manual of mental disorders: DSM-5. 5. Washington, DC: American Psychiatric Publishing; 2013.

2 McClain MB, Hasty Mills AM, Murphy LE. Inattention and hyperactivity/impulsivity among children with attention-deficit/hyperactivity-disorder, autism spectrum disorder, and intellectual disability. Res Dev Disabil. 2017 Nov; 70:175-84.

3 Miodovnik A, Harstad E, Sideridis G, Huntington N. Timing of the diagnosis of attention-deficit/hyperactivity disorder and autism spectrum disorder. Pediatrics. 2015 Oct; 136(4):e830-7.
4 Ros R, Graziano PA. Social functioning in children with or at risk for attention deficit/ hyperactivity disorder: a meta-analytic review. J Clin Child Adolesc Psychol. 2018 Mar; 47(2):213-35.

5 Luteijn EF, Serra M, Jackson S, Steenhuis MP, Althaus M, Volkmar F, et al. How unspecified are disorders of children with a pervasive developmental disorder not otherwise specified? A study of social problems in children with PDD-NOS and ADHD. Eur Child Adolesc Psychiatry. 2000 Sep;9(3): 168-79.

6 Mulligan A, Anney RJL, O’Regan M, Chen W, Butler L, Fitzgerald M, et al. Autism symp- toms in attention-deficit/hyperactivity disorder: a familial trait which correlates with conduct, oppositional defiant, language and motor disorders. J Autism Dev Disord. 2009 Feb; 39(2):197-209.

7 Nijmeijer JS, Hoekstra PJ, Minderaa RB, Buitelaar JK, Altink ME, Buschgens CJM, et al. PDD symptoms in ADHD, an independent familial trait? J Abnorm Child Psychol. 2009 Apr;37(3):443-53.

8 Okyar E, Görker I. Examining the autistic traits in children and adolescents diagnosed with attention-deficit hyperactivity disorder and their parents. BMC Psychiatry. 2020 Jun; 20(1):285. 
9 Reiersen AM, Constantino JN, Volk HE, Todd RD. Autistic traits in a populationbased ADHD twin sample. J Child Psychol Psychiatry. 2007 May;48(5):464-72.

10 Salley B, Gabrielli J, Smith CM, Braun M. Do communication and social interaction skills differ across youth diagnosed with autism spectrum disorder, attention-deficit/hyperactivity disorder, or dual diagnosis? Res Autism Spectr Disord. 2015 Dec;20:58-66.

11 Antshel KM, Russo N. Autism spectrum disorders and ADHD: overlapping phenomenology, diagnostic issues, and treatment considerations. Curr Psychiatry Rep. 2019 22; 21(5):34.

12 Mikami AY, Miller M, Lerner MD. Social functioning in youth with attention-deficit/ hyperactivity disorder and autism spectrum disorder: transdiagnostic commonalities and differences. Clin Psychol Rev. 2019;68:54-70.

13 Rommelse NN, Geurts HM, Franke B, Buitelaar JK, Hartman CA. A review on cognitive and brain endophenotypes that may be common in autism spectrum disorder and attention-deficit/hyperactivity disorder and facilitate the search for pleiotropic genes. Neurosci Biobehav Rev. 2011 May;35(6):1363-96.

14 Rao PA, Landa RJ. Association between severity of behavioral phenotype and comorbid attention deficit hyperactivity disorder symptoms in children with autism spectrum disorders. Autism. 2014 Apr;18(3):272-80.

15 Schwenck C, Freitag CM. Differentiation between attention-deficit/hyperactivity disorder and autism spectrum disorder by the Social Communication Questionnaire. Atten Defic Hyperact Disord. 2014 Sep;6(3):221-9.

16 Sprenger L, Bühler E, Poustka L, Bach C, Heinzel-Gutenbrunner M, Kamp-Becker I, et al. Impact of ADHD symptoms on autism spectrum disorder symptom severity. Res Dev Disabil. 2013 Oct;34(10):3545-52.

17 Yerys BE, Wallace GL, Sokoloff JL, Shook DA, James JD, Kenworthy L. Attention deficit/hyperactivity disorder symptoms moderate cognition and behavior in children with autism spectrum disorders. Autism Res. 2009 Dec;2(6):322-33.

18 Ghaziuddin M, Welch K, Mohiuddin S, Lagrou R, Ghaziuddin N. Utility of the social and communication questionnaire in the differentiation of autism from ADHD. J Dev Phys Disabil. 2010 Aug;22(4):359-66.

19 Kochhar P, Batty MJ, Liddle EB, Groom MJ, Scerif G, Liddle PF, et al. Autistic spectrum disorder traits in children with attention deficit hyperactivity disorder. Child Care Health Dev. 2011 Jan;37(1):103-10.

20 Mouti A, Dryer R, Kohn M. Differentiating autism spectrum disorder from ADHD using the social communication questionnaire. J Atten Disord. 2019 Jun;23(8):828-37.

21 Lord C, Rutter M, DiLavore PC, Risi S. Autism diagnostic observation schedule (ADOS). Los Angeles, CA: Western Psychological Services; 1999.

22 Lord C, Rutter M, Le Couteur A. Autism diagnostic interview-revised: a revised version of a diagnostic interview for caregivers of individuals with possible pervasive developmental disorders. J Autism Dev Disord. 1994 Oct;24(5):659-85.

23 Grzadzinski R, Dick C, Lord C, Bishop S. Parent-reported and clinician-observed autism spectrum disorder (ASD) symptoms in children with attention deficit/hyperactivity disorder (ADHD): implications for practice under DSM-5. Mol Autism. 2016 Dec;7(1):7.

24 Banaschewski T, Hollis C, Oosterlaan J, Roeyers $\mathrm{H}$, Rubia K, Willcutt E, et al. Towards an understanding of unique and shared pathways in the psychopathophysiology of ADHD. Dev Sci. 2005 Mar;8(2):132-40.

25 Tye C, Asherson P, Ashwood KL, Azadi B, Bolton P, McLoughlin G. Attention and inhibition in children with ASD, ADHD and comorbid ASD + ADHD: an event-related potential study. Psychol Med. 2014 Apr;44(5): 1101-16.

26 Tye C, Johnson KA, Kelly SP, Asherson P, Kuntsi J, Ashwood KL, et al. Response time variability under slow and fast-incentive conditions in children with ASD, ADHD and ASD+ADHD. J Child Psychol Psychiatry. 2016 Dec;57(12):1414-23.

27 Tye C, Mercure E, Ashwood KL, Azadi B, Asherson P, Johnson MH, et al. Neurophysiological responses to faces and gaze direction differentiate children with ASD, ADHD and ASD + ADHD. Dev Cogn Neurosci. 2013 Jul; 5:71-85.

28 Tye C, Battaglia M, Bertoletti E, Ashwood KL, Azadi B, Asherson P, et al. Altered neurophysiological responses to emotional faces discriminate children with ASD, ADHD and ASD+ADHD. Biol Psychol. 2014 Dec;103: 125-34.

29 Banaschewski T, Neale BM, Rothenberger A, Roessner V. Comorbidity of tic disorders \& ADHD: conceptual and methodological considerations. Eur Child Adolesc Psychiatry. 2007 Jun;16(Suppl 1):5-14.

30 Burstein M, Ginsburg GS, Tein JY. Parental anxiety and child symptomatology: an examination of additive and interactive effects of parent psychopathology. [corrected]. J Abnorm Child Psychol. 2010 Oct;38(7):897-909.

31 Roessner V, Becker A, Banaschewski T, Rothenberger A. Psychopathological profile in children with chronic tic disorder and coexisting ADHD: additive effects. J Abnorm Child Psychol. 2007 Feb;35(1):79-85.

32 Kamp-Becker I, Poustka L, Bachmann C, Ehrlich S, Hoffmann F, Kanske P, et al. Study protocol of the ASD-Net, the German research consortium for the study of Autism Spectrum Disorder across the lifespan: from a better etiological understanding, through valid diagnosis, to more effective health care. BMC Psychiatry. 2017 Dec;17(1):206.

33 World Health Organization. The ICD-10 classification of mental and behavioural disorders: clinical descriptions and diagnostic guidelines. Geneva: World Health Organization; 1992.
34 Rühl D, Bölte S, Feineis-Matthews S, Poustka F. ADOS Diagnostische Beobachtungsskala für Autistische Störungen. Bern: Verlag Hans Huber; 2004.

35 Rutter M, Le Couteur A, Lord C. Autism Diagnostic InterviewTM, Revised. Los Angeles, CA: Western Psychological Services; 2003.

36 Bölte S, Rühl D, Schmötzer G, Poustka F. ADI-R Diagnostisches Interview für Autismus-Revidiert. Deutsche Fassung des Autism Diagnostic Interview-Revised von Michael Rutter, Ann LeCouteur und Catherine Lord. Bern: Hogrefe AG, Verlag Hans Huber; 2006.

37 American Psychiatric Association. Diagnostic and statistical manual of mental disorders DSM-IV-TR (Text Revision). 4th ed. Washington: APA; 2000.

38 Bölte S, Poustka F. Diagnostische Beobachtungsskala für Autistische Störungen (ADOS): Erste Ergebnisse zur Zuverlässigkeit und Gültigkeit. Z Für Kinder- Jugendpsychiatrie Psychother.. 2004 Jan;32(1):45-50.

39 Poustka F, Lisch S, Rühl D, Sacher A, Schmötzer G, Werner K. The standardized diagnosis of autism, autism diagnostic interview-revised: interrater reliability of the German form of the interview. Psychopathology. 1996;29(3):145-53.

40 van der Meer JMJ, Oerlemans AM, van Steijn DJ, Lappenschaar MGA, de Sonneville LMJ, Buitelaar JK, et al. Are autism spectrum disorder and attention-deficit/hyperactivity disorder different manifestations of one overarching disorder? Cognitive and symptom evidence from a clinical and population-based sample. J Am Acad Child Adolesc Psychiatry. 2012 Nov;51(11):1160-72.e3.

41 Visser JC, Rommelse NN, Greven CU, Buitelaar JK. Autism spectrum disorder and attention-deficit/hyperactivity disorder in early childhood: a review of unique and shared characteristics and developmental antecedents. Neurosci Biobehav Rev. 2016 Jun;65: 229-63.

42 Willcutt EG, Nigg JT, Pennington BF, Solanto MV, Rohde LA, Tannock R, et al. Validity of DSM-IV attention deficit/hyperactivity disorder symptom dimensions and subtypes. J Abnorm Psychol. 2012 Nov;121(4):991-1010.

43 Wood-Downie H, Wong B, Kovshoff H, Cortese S, Hadwin JA. Research Review: A systematic review and meta-analysis of sex/gender differences in social interaction and communication in autistic and nonautistic children and adolescents. J Child Psychol Psychiatry. 2021;62(8):922-36.

44 Poustka L, Rühl D, Feineis-Matthews S, Poustka F, Hartung M, Bölte S. Diagnostische Beobachtungsskala für Autistische Störungen-2 (ADOS-2). Bern: Verlag Hans Huber; 2015. Deutschsprachige Fassung der Autism Diagnostic Observation Schedule, Second Edition (ADOS-2) C. von Lord, M. Rutter, P.C. Di-Lavore, S. Risi, K. Gotham und S.L. Bishop (Teil I: Module 1 bis 4) und C. Lord, R.J. Luyster, K. Gotham und W. Guthrie (Teil II: Kleinkind-Modul). 